This item was submitted to Loughborough's Research Repository by the author.

Items in Figshare are protected by copyright, with all rights reserved, unless otherwise indicated.

\title{
Morphological and biological characterization of density-engineered foams fabricated by ultrasonic sonication
}

PLEASE CITE THE PUBLISHED VERSION

http://dx.doi.org/10.1007/s10853-010-4944-z

\section{PUBLISHER}

(c) Springer

VERSION

AM (Accepted Manuscript)

\section{PUBLISHER STATEMENT}

This work is made available according to the conditions of the Creative Commons Attribution-NonCommercialNoDerivatives 4.0 International (CC BY-NC-ND 4.0) licence. Full details of this licence are available at: https://creativecommons.org/licenses/by-nc-nd/4.0/

\section{LICENCE}

CC BY-NC-ND 4.0

\section{REPOSITORY RECORD}

Torres-Sanchez, Carmen, and Jonathan R. Corney. 2019. "Morphological and Biological Characterization of Density-engineered Foams Fabricated by Ultrasonic Sonication". figshare. https://hdl.handle.net/2134/22629. 


\title{
Morphological and biological characterization of density engineered foams fabricated by ultrasonic sonication
}

\author{
C. Torres-Sánchez ${ }^{*}, 1$, J. R. Corney ${ }^{2}$ \\ ${ }^{1}$ Department of Mechanical Engineering, School of Engineering and Physical \\ Sciences, Heriot-Watt University, Edinburgh, EH14 4AS, UK \\ ${ }^{2}$ Department of Design, Manufacture and Engineering Management, University of \\ Strathclyde, Glasgow, G1 1XJ, UK \\ Phone: +44 (0) 1314513531 \\ E-mail: c.torres@hw.ac.uk
}

\begin{abstract}
The successful manufacture of functionally tailored materials (e.g. density engineered foams) for advanced applications (e.g. structures or in bioengineering) requires an effective control over the process variables. In order to achieve this, density gradation needs to be represented and quantified. Current density measurement techniques offer information on bulk values, but neglect local position as valuable information (i.e. do not associate density scalar values with specific location, which is frequently critical when mechanical properties or functionalities have to be engineered). In this paper, we present a method that characterizes the density gradation of engineered foams manufactured by the sonication technique, which allows the generation of sophisticated porous architectures beyond a simple linear gradient. A 3D data capture $(\mu \mathrm{CT})$ and a flexible analysis software program (ImageJ) are used to obtain 'global' density gradation values that can, ultimately, inform, control and optimize the manufacture process. Polymeric foams, i.e. polyurethane (PU) foams, were used in this work as proof of concept. The measurements performed on the PU foams were validated by checking consistency in the results for both horizontal and vertical image slices. Biological characterization was done to assess the samples' tailored structure viability as scaffolds for tissue engineering. The comparison between untreated and sonicated samples yielded a $12.7 \%$ of increment in living cell count adhered to the walls after treatment. The conclusions drawn from this study may inform the design and manufacture of density engineered materials used in other fields (e.g. structural materials, optoelectronics, food technology, etc)
\end{abstract}

\section{Keywords}

FGM, density gradient, sonication, characterization, $\mu C T$, ImageJ, biomimicry. 


\section{Introduction}

Functionally graded and tailored materials (FGMs) are those designed and manufactured in such a way that their properties vary within the bulk to match the functionalities required for a given application. Density engineered foams are an example of these FGMs where, instead of blending different materials, the material itself can have its properties modified by creating voids. Such controlled distribution can affect its mechanical, chemical, thermal or acoustic properties, providing tailored functionalities. Solid foams with tailored density hold synergistic features that make them a sought-after material in a myriad of fields. Examples of foams engineered for advanced applications are: structural materials with use in aerospace, automobile, construction, etc (where maximum strength is obtained with a minimal amount of weight); and engineered 3D structures with a controlled degree of interconnectivity, so they can be used as bone scaffolds in tissue engineering and orthopedics or in filtration membranes. Foams with a controlled density are also becoming important in other industries such as food technology, where micro-architecture of the foam has a direct impact on sensory properties of food [1], optoelectronics to guide magnetic, electrical fields or light and minimize energy loses [2], and microelectronics to produce minimum thermal conductivity [3]. The characterization of the density distribution in these solid foams is of vital importance because this data can be used to inform the manufacture process and further improve their properties and functionalities. However, the manufacture of functionally tailored materials (e.g. density engineered foams) has been developed at a faster pace than their effective representation and analysis methods. Consequently, a standard methodology for the $3 \mathrm{D}$ characterization and quantification of density gradient materials is still missing.

This paper describes a procedure used for the measurement, representation and quantification of density tailored polymeric foams manufactured by the sonication method. The next section (and subsections 2.1 and 2.2) contains an explanation of this novel fabrication technique for density tailoring of foams and contrasts it with other manufacturing processes currently (or potentially) used to produce engineered foams. The subsequent section (section 3) will introduce morphological density characterization methods for density tailored materials and 
also includes an explanation of the methodology used in this work. A set of results obtained using this method is shown in the subsections of section 3 as an example of the capabilities and versatility of this characterization method. Biocompatibility assessment and its results, motivated by an interest in using these materials as orthopedic implants, are shown in section 4. In order to complete the study, the results are validated against visual inspection (due to the lack of a standard procedure for the quantification of 'global' gradients) and a discussion on the veracity of the analysis will follow (section 5). Finally, conclusions (section 6) from this study will be drawn and a translation to the design and manufacture of other density tailored materials will be highlighted.

\section{Manufacture of porosity tailored materials}

Despite the need for functionally tailored, or heterogeneous, materials in current technology markets, manufacturing processes lag behind this demand. This is because traditional technologies to produce homogeneous materials are fully embedded in current industrial manufacturing systems. Attempts are being made to change the industrial adherence to producing only homogeneous materials and several successful manufacturing strategies are now working laboratory processes. Some of the most promising and viable examples are presented later in this section. A complete change in mindset has not yet occurred because the new procedures have not been around for long enough or have not been fully implemented for mass production at industrial scale.

In particular, density graded foams are an example of heterogeneous materials, i.e. their texture is non-homogeneous. They are also an example of FGMs, because the distribution of the two phases (material and void) is 'ad-hoc', distributed to achieve a specific functionality, e.g. providing optimized acoustic, thermal, vibration or impact damping. From a commercial viewpoint, these materials' manufacturing methods have been limited to the mass-production of homogeneous foams by the reaction injection molding, i.e. high pressure extrusion method [4]. If the full advantages of a tailored micro-architecture are to be exploited, new manufacturing processes that allow porosity engineering of the structure have to be proposed. 
The following subsection presents selected examples of manufacturing methods for porosity graded foams (of polymeric, ceramic, metallic or composite nature).

\section{Emerging techniques for the manufacture of porosity tailored materials}

\section{Layer Manufacturing by Rapid Prototyping technologies.}

In this method, 3D-CAD data representation is used to fabricate solid physical models via the deposition of powder material (polymer, metal, ceramic or a mixture) in a bottom-up layer-by-layer building sequence previously programmed on a PC that drives the system. This is a building sequence, previously programmed on a PC that drives the system. Once the particles have been laid on a rising worktop, they undergo laser sintering, fusing or gluing [5]. This technology offers benefits such as reduction of manufacture time, low cost of prototyping and, in most cases, no need for special tooling to obtain a net-shape object [6]. 3D porous structures with a graded window-size have been fabricated (Figure 1a) using layer manufacturing technology. However, the intrinsic morphology of those structures, with contra-naturam straight edges [7], limits their applications. For example, this is not the best approach for the design and fabrication of porous scaffolds for tissue engineering or lattices [8], where the ratio of strength to weight has to be maximized. From a biomimicry perspective, natural porous structures (e.g. plant stems, tree truck, bones, feathers and bills) are designed by nature in such a way that strength is maximized. By contrast, in rapid-prototyped structures stiffness is the optimized variable (Figure 1b). If a biomimetic approach is to be considered in order that nature's lessons on optimization of lightweight structures can be harnessed for engineering solutions, porous materials that resemble natural structures have to be fabricated.

\section{Gel casting and freeze casting}

This technique, broadly used in the production of tissue engineering scaffolds, is a sophisticated fabrication process that involves multiple steps. Initially, a mold has to be manufactured. It will support the solidifying pattern of a second material, so it is a sacrificial structure. This temporary scaffold can be made of gel [9] or, most 
recently, freezing water has been used as a way of controlling the shape and geometry of the structure [10] [11]. In both cases, this template is impregnated with a slurry (i.e. water mixed with powder) and left to dry (or freeze-dry). The powder occupies the empty spaces and it then forms a 'green body'. This porous artifact is not sufficiently strong for almost any application. Therefore, a further step of sintering follows, when the sacrificial network disappears and the powder fuses to form a stronger matrix. Despite the laboriousness of the method, Deville et al. have managed to synthesize porous hydroxyapatite (a ceramic) scaffolds with higher compressive strength than other scaffolds of similar porosity reported in the literature [11].

\section{Controlled 'bubble forming' techniques}

In general terms, these techniques are based on producing and stabilizing bubbles within a mass. The bubbles can be created by gas by-products of the chemical reaction or injected externally. Ceramic foams [12], polymeric foams [13] and metal foams [14] have been manufactured via this method which does not provide a high control on the porosity gradation per se. If engineered foams are intended, a better control on the gas injection, or production, is needed. This implies the control of the source for that aeration. It is easier when the gas is injected externally, but when the gas is created as a by-product of the chemical reaction, the task is more challenging.

Laser foaming has emerged as an alternative process to control the heat source that releases the blowing agents from the mixture [15]. In a preparatory step, metal powder and foaming agent are mixed. Foaming is initiated by heating up to the mixture's melting point which produces a unidirectional expansion. Porosity gradation can be controlled by the laser irradiation power, the dwell time and the cooling rate of the molten material. This is a good example of how the physical blowing of the mixture can be controlled to produce engineered cellular materials. These methods' main limitations are the linearity of the porosity gradation, the erratic growth of the bubbles, and the coalescence or deformation of the generated pores if the processing conditions are not properly adjusted, especially at very fast processing speeds. The latter is not a trivial issue, because the processing speed has to counteract the gravitational effects of the molten metal. Once these 
drawbacks are addressed, the method presents itself like a potential alternative to the manufacture of metal and composite foams with engineered structures.

\section{Replica Processing for the engineering of structural foams}

The most frequently used method for engineering structural foams is the patented 'Schwartzwalder and Somers' [16] replica process. Initially used for the fabrication ceramic structures only, it is also a versatile technique used for metal foams [17]. The main strength in this manufacturing strategy lies in the possibility to create almost any porosity gradation within the final material. Highly controlled cellular structures can be used for the 'replica process' and there are some manufacturing examples already: ceramic [18], metal [19], and composite materials [20] in order to obtain synergistic properties from the blend of two different materials (e.g. chemical properties of a ceramic and mechanical properties of a polymer). This technique uses a foamed polymer as a pre-form for the final porous material. The polymeric pre-form is soaked in a slurry of ceramic, metal, polymer or composite, and sintered at elevated temperatures with or without increasing pressure conditions. When the sacrificial network decomposes, the obtained structure presents a similar porous structure, so by engineering the porosity gradation in the pre-form, the final material is fit for purpose, with the interconnectivity, structural features required for a specific application. The 'replica process' produces foams with predominantly 'open pores', which is ideal for biomedical applications or where permeability is an important issue, but has the drawback that pyrolysis of the polymer skeleton in many cases produces structural flaws or leaves residues in the final cellular material. In addition, because of the difference in thermal behavior while sintering between the preform polymer and the final material, some dimensional shrinkage will occur during fabrication.

\section{Tailoring microstructure and functionalities by adding particles}

The addition and blend of reactive or inert particles to the foaming mixture offers the possibility to create ad-hoc pore sizes and porosity gradation. Foams have been manufactured with the incorporation of electrically-, thermally-, and magnetically-active fillers [21]. These foams have exhibited superior mechanical properties when compared to those manufactured using the replica technique 
solely. Other porogen particles, such as salt or granules, have been added to sintered or hot pressed mixtures with the intention of creating interconnected pores when these dissolved or were washed out. Inert particles whose function is the creation of voids or channels have been long employed in the fabrication of scaffolds for tissue regeneration [22], where a large interconnectivity degree is desired.

In those cases where the chemical composition of the porous specimen or its toxicity levels can be an issue that restrains from its broad use in some applications, some of the methods presented above may not be appropriate (e.g. bubble-forming techniques controlled by surfactants, or the addition of certain porogen particles). The next section surveys a novel method recently presented that allows the tailoring of structures with graded porosity by acoustically-aided

foaming. No external chemicals, particles or intermediate processes are necessary, therefore this technique is highly prescribed in those applications where hygienic conditions and non-contact processing is required to prevent the samples from cross-contamination. In addition, the possibility of tailoring sophisticated porosity gradations with this technique, rather than simply linear gradients, shows an added value when a pre-form foam is designed and tailored to use in the 'replica processing' technique, aforementioned.

\section{Generation of porosity tailored foams by sonication}

In this method, first reported in 2008 [23], a polymeric melt undergoing foaming is irradiated using low power ultrasound in the range of 20-30kHz. By controlling the acoustic field that the polymerizing mixture is subject to, maxima and minima of energy (i.e. antinodes and nodes in the acoustic standing wave) can be manipulated and imposed on the viscoelastic foam, so once this solidifies, the pore size will be graded along two or three dimensions of the matrix. The underpinning principle of this effect is the 'stable cavitation' phenomenon. The bubbles in the polymeric matrix are set to resonate in the acoustic field, which produces a volumetric growth due to the alternate expansion and compressions of the bubbles that pump the dissolved gas in the matrix into their cavities [24]. 


\section{Manufacture of density tailored polymeric foams in this study}

In this work, the sonication technique has been employed to manufacture porosity graded polyurethane foams. The monomers employed for this reaction were MDI and polyether polyol (Dow Europe GmbH, Switzerland), and distilled water which played a role as a catalyst. The reaction of the monomers with water produces polyurethane and gas carbon dioxide, blowing agent for the melt. All mixtures were sonicated in an open-vessel container and no vacuum or pressure was applied during the casting or curing of the foamed cylinders. This was done to avoid the build-up of the internal pressure due to the water vapor and gases generated by the reaction that could provoke unwanted implosion of bubbles. Temperature and conductivity probes were employed to monitor the development of the reaction and detect stage changes, so the ultrasound could be irradiated when most favorable. Figure 2 shows a schematic of the experimental rig. Further details on the experimental process for the sonication of the samples and details on the detection of the different polymerization stages can be found elsewhere [25-27].

In order to obtain a better accuracy with this process and control over more sophisticated porous structures, a morphological characterization of the porosity gradation has to be performed. The results from this characterization (i.e. pore size, pore distribution and its location) will be used to feedback the manufacturing process for an improved engineering of the porous micro-structure. The next section presents work on the characterization strategy followed for this work on porosity tailored polymeric foams.

\section{Morphological characterization of density gradation in the polymeric foams}

Morphological characterization of solid foam micro-structure is not trivial and many attempts have been made to quantify and represent porosity gradation in these engineering foams. This section describes the methodology used to couple porosity characterization and location in the polymeric foams with tailored porosity under study. Prior to that, we present a compilation of current solutions 
applied to the measurement of porosity graded structures that were considered in our work but were discarded when considered unsuitable for this application for the reasons described below. This demonstrates the lack of standardization in the methodology for 3D characterization and quantification of density engineered materials.

Having described the graded properties of these foams, it is obvious that traditional porosity measurement methods such as Archimedes', mercuryporosimetry or infiltration methods are not sufficient for the complete characterization of these materials, especially when the interconnectivity in these materials may vary (open, connected pores or closed cavities). The reason is that they relay bulk, global values, when the local property is the interesting one. There are many software packages available for image analysis and normally these were prepared for different functions. Because of the distinctive aims of each strategy, there is not a standardized one-for-all method, but several 'fit-forpurpose' applications. Those considered in this study are listed below, along with the limitations that led to their discharge for this specific application. Traditional methods such as the 'PPI method' (i.e. pores per inch) and, more recently Visiocell ${ }^{\circledR}$ were disregarded because these methods can be uncertain, with poor reproducibility and heavily dependent on users' input [28]. In the 'PPI method', number of pores is counted over a 1 inch-long random line drawn on images of cross-sections. The Visiocell ${ }^{\circledR}$ method also uses images of crosssections and user's input to select a single cells. Once that is done, the program calculates parameters of the foam such as pore diameter, density, etc. These two methods assume a uniform distribution of quasi-spherical pores, hence its limitation for heterogeneous applications.

FoamView [29] was compiled with an intention to analyze real 3D polymeric foams. FoamView extracts structural information from the anisotropic foam (e.g. strut length, window area, cell volume, surface area, etc) and quantifies its cell shape diversity. The latter is an interesting quality when working with heterogeneous foams. The great potential in this method is diminished by the lack of topographic information for these features. While FoamView is a robust method for obtaining information on cell features and shape, it does not couple it with location, so the results cannot be interpreted in a three-dimensional space. 
The Topo-porosity tool [26], created in MatLab, presents the advantage of coupling both porosity value and location. It uses isoline maps that join points of equal porosity with a line, like topographic maps joining points of equal altitude. This strategy allows the representation and analysis of porosity graded structures and couples both values and position in the polymeric matrix. The main drawback for the Topo-porosity tool is that the representation of porosity gradation is graphical, not scalar.

Image Pro-Plus [30] is an example of the standard methodology of many commercial image analysis software packages available. Using 2D images the user can choose 'active' areas and select the pores on it. The program conveys values for a myriad of features such as perimeter, density, diameter, etc. Image Pro-plus in particular offers information on the shape of the pores (i.e. spheroidicity) using values of maximum and minimum diameter. As in most of the other commercial programs with similar functionalities, its main disadvantages are the manual user input and the poor segmentation which produces that small pores are left out of the analysis.

In the search for an image processing system that includes the smallest pore sizes and detects the thinner cell walls that otherwise are neglected, Malcolm et al. [29] developed an automated image processing method for metal foams with the remarkable advantages of providing a better segmentation over commercially available softwares and more speed (x10) over manual segmentation. This method allows characterization of 2D slices using individual parameters (e.g. aspect ratio, compactness) and group parameters (e.g. ratio of void area to total area -porosity-, distribution of the pore sizes with respect to the frequency of occurrences and distribution of pore sizes with respect to the distance from the centre of the foam). Although the individual parameters are reported in scalar numbers, the group parameters can only be represented in graphical form and this is a drawback for the work intended here. The most interesting parameter (i.e. distribution of the pore sizes with respect to the centre) lacks versatility for the purpose of this study. This is because pore distribution across the cross-section is not necessarily symmetrical or centered on the vertical axis but often skewed to one side [31].

In the absence, to the author's knowledge, of a systematic criterion that can automate the characterization of density gradient in a $3 \mathrm{D}$ volume, a compromised 
solution was adopted. It involved the aggregation of a highly efficient data capture $(\mu \mathrm{CT})$ and a flexible analysis software (ImageJ). Computer micro-tomography was used because it is a non-destructive technique that allows precise viewing of the cellular structure within a 3D volume. Different regions in the sample were identified (i.e. local properties) and the characterization was done using ImageJ, which was chosen in this study for its robustness and versatility. Local properties were integrated and analyzed further in order to generate both visual and quantitative account of the global density gradation within the porous sample. Further details can be found in the following section.

\section{Methodology}

A micro computer tomography scanning $(\mu \mathrm{CT})$ system was used to obtain highdefinition horizontal cross-section images of the foams. The specimens were scanned at $0.2 \mathrm{~mm}$ pixel resolution and angular increment of -0.1353 (relaying 2661 projection images per specimen). For the reconstructed volume of the sample shown in Figure 3, 942 by 942 by 838 voxels were obtained at a voxel size of $0.1065 \mathrm{~mm}^{2}$. The number of images produced was 658 . From these, 43 at the top and 162 at the bottom were discarded because those were either out of the sonication field, and therefore shown different features to the sonicated foams (e.g. skin around the perimeter of the foam), or suffered from 'wall effects' (i.e. presented deformed pores). The final number of images to analyze was 452 , starting from the top of the sample (slice \# 1), to the bottom (slice \# 452).

The same regions of interest (ROIs) were selected in each of the cross-sections of the foam, as shown in Figure 4. The morphological characterization of those regions was done using ImageJ (W. Rasband, 1997, National Institutes of Health, USA), a java-based open-source software for image processing and analysis broadly used in medical imaging, microscopy and material sciences [32]. Thresholding of the images, the most important stage in image analysis [28] was a simplified task in this case. Thresholding, employed as a segmentation technique, involves the distinction between those voxels which represent material and those which represent void. The white pixels of the foams shown against the black solid 
background allowed an easy binarization, and noise was kept to a minimum in all the evaluations.

\section{Characterization methodology of the 2D image stack: key parameters}

ImageJ allows the systematic extraction of key parameters values such as mean grey value, maximum and minimum grey value, centre of mass, area, perimeter, standard deviation, circularity, centroid, skewness, kurtosis, etc. For the purpose of this work, mean grey value and skewness were studied.

'Mean grey value' is defined as the average grey value within the selected region. This is the sum of the grey values of all the pixels in the selection divided by the number of pixels. The ImageJ reported value had to be calibrated, so the results could be comparable within the same stack and the different regions of interest. Once standardized, the $\%$ density could be drawn, i.e. knowing the maximum grey value on each slice, the density (rho) was calculated in each case as follows:

$$
\rho(\%)=\frac{\text { mean grey value }}{\text { maximum grey value }} \cdot 100
$$

Density (in \%) resulted to lie in the range [4-14\%] and was plotted against number of slice to determine the variation rate throughout the sample, on each ROI's vertical axis. Figure 5 shows ROI density variation as an exemplar of this process. Best fitting was applied to each ROI and cubit equations were found to best match with the lowest residual (i.e. not larger than $|-1|$ at any point).

$$
y(x)=p_{1} \cdot x^{3}+p_{2} \cdot x^{2}+p_{3} \cdot x+p_{4}
$$

Table 1 lists the $\mathrm{p}_{\mathrm{i}}$ coefficients for the 9 ROIs.

\section{Characterization methodology of the $3 D$ volume}

The study of the porosity gradation was done by merging the local results obtained from the 9 ROIs, as defined in the previous section. Visualization of the global density gradation within the sample was reached by 'unrolling' the cylinder formed once all the local densities from each ROI on their vertical axis were displayed in columns (Figure 6). Starting at the central column (ROI 1) and continuing with 2 , to 9 in anticlockwise, the 'unrolled' cylinder was made 'flat', 
metaphorically speaking, and the densities for each slice were plotted to show gradients (Figure 7). ROIs 2, 3, 4, 5 and 6 present the largest gradients (i.e. slope) between slices \#120-400. This is translated to a density gradient on the OX axis, from low density to higher.

'Skewness' is the dimensionless coefficient of symmetry (third order of the spatial moment, parameters derived from $2^{\text {nd }}$ moments [33]). As per its definition, when value is zero, there is symmetric distribution. When the coefficient of skewness is $<0$, the distribution is asymmetric to the left (i.e. tail extends left of the centre of mass; i.e. tilted to the right), and when $>0$, the asymmetry is to the right (i.e. tail extends right of the centre of mass; i.e. tilted to the left).

The coefficient of skewness values in this sample were in the range, [2.28 - 8.53], confirming the positive asymmetric distribution.

\section{Validation}

To demonstrate that the results of the areas selected as the ROIs (on the horizontal slices) represent a variation of porosity within the foam, a comparison of these against vertical cross sections of the same foam was performed (Figure 8).

These figures show a variation in the porosity values for both horizontal and vertical slices. The variable on the 'OY' axis is slice \#number, and on ' $O X$ ' the different ROIs, as per section under study. For example, the density values for slice \#200 on the top left panel reads that ROI 3 has a density of $10.5 \%$, ROI 2 $7.8 \%$ and ROI 9 approx $7.8 \%$. This informs that the transition from ROI 3 to ROI 2 has a gradient in density, but from ROI 2 to ROI 9 there is none, i.e. the density values remain constant. From these graphs it can be observed that the regions ROI 2, ROI 3, ROI 4 and ROI 5 have the largest gradient of density. Therefore, these should be the zones where the pore sizes differ the most when compared to the rest of the foam's bulk.

Visual inspection on the foam's vertical cross sections shows that the areas containing ROIs 3, 4 and 5 are the one that shows a higher density gradient, with larger changes in the density values. It can be seen on Figure 9 that ROIs 1 and 4 
have larger pore sizes, which is consistent with the results in Figure 8 (i.e. horizontal global density). Figure 9 (left) also shows positive coefficient of skewness (i.e. asymmetric distribution to the right, tilted to the left), which is consistent with the numerical values extracted with the image analysis program.

\section{Biological characterization of the porosity-tailored foams}

With the intention of assessing the use of these porosity tailored structures as potential tissue engineering scaffolds, and how the porosity distribution may impact on this feasibility, biological characterization was attempted. To test the structure's suitability, MC3T3-E1 osteoblast-like cells were cultured on the foams and examined for viability using AlamarBlue ${ }^{\circledR}$, a cell health indicator used in cytotoxicity assays (Figure 10a and c) where pink color indicates metabolic active cells. The preosteoblastic cells' fluorescence response was then captured. Living cells were stained green and dead cells were red when visualized under a confocal scanning microscope (Figure 10b and d). A higher viability was observed for the adherent cells on the porosity graded foam manufactured by sonication, in comparison to those adhered on the homogeneous foams. Figure 10 shows the distribution of cells on the surface on the scaffolds. Image analyses convey a mean increment of $12.7 \%$ of adhered living cells when comparing the images from the seeded treated foam versus the seeded homogeneous samples. These results reveal the suitability of the porosity tailored foams as a substrate for tissue engineering. Further analyses are needed to establish whether the coupled effect of porosity gradation with pore size has an implication on the favorable environment for the cells to proliferate, versus that created in the homogeneous foam.

\section{Discussion}

The need for a systematic way of measuring and representing global density gradation has driven this novel approach, where $\mu$-CT binarized images were analyzed using ImageJ, a versatile software program. This procedure allowed the 
characterization of density engineered foams that were manufactured using the sonication process: a polymeric melt undergoing foaming was irradiated with ultrasound at 'sensitive' formation stages, controlling the amount of gas being pumped into the bubbles which, once solidified, formed cavities.

Local density values have been traditionally measured, but strategies to establish 'global' gradients of density are not that well established. This parameter is important for the optimization and full exploitation of density tailored materials that are involved in many applications such as in aerospace, automotive, building construction, filtration, acoustic, thermal and vibration damping, food technology, optoelectronics, etc.

Using this technique (aggregation and coupling of $\mu$-CT and ImageJ), the 'global' gradient results were plotted using an 'unrolling' action of the ROIs' values compilation. The derived data from the coefficients of skewness, cubic fitting coefficients, global gradients, and local gradients of the 9 ROIs in their vertical axis were coherent, and depicted ROIs 2, 3, 4 and 5 as the areas with the highest density gradation (Figure 7), as expected.

This result is consistent with the visual inspection done on the foam (Figure 9), where the same regions hold the bell-shape distribution of bigger pore sizes. The $\mathrm{p}_{\mathrm{i}} \mathrm{s}$ coefficients from the cubic fitting listed in Table 1 indicate that ROIs 1, 2, 3 , and 4 have the same trend for the density gradation in those regions. This is also consistent with the previous statements.

In summary, ROIs 1-6 are the areas in the foam that present higher gradient of density (i.e. larger changes in density values from region to region). This can be seen by visual inspection (Figure 9) and using the global gradient map (Figure 7). This result was expected as it is consistent with the manufacturing data, because the sample was fabricated so it would show a bell-shaped distribution of density centered at ROI 1 and skewed towards ROI 4 (i.e. the sample was placed at an antinode of acoustic pressure within the acoustic field) [31].

Biological viability was tested in these samples manufactured by sonication in order to assess their potential application as scaffolds in bioengineering. The 
promising results shown here (i.e. large $\%$ of metabolic cells alive after the incubation process in the sample manufactured by sonication) demonstrate that this technique is a feasible biomimetic solution for the manufacture of medical devices. Further research is needed to examine the independent influence of the material by itself and the sonication-aided manufacturing technique on the biological viability. A successful bio-compatibility case is considered to be multifactorial (i.e. several surface characteristics such as surface morphology and chemical functionality, govern the extent of cell adhesion and proliferation, etc). The contribution offered by the sonication method to this positive biocompatibility result remains to be quantified.

\section{Conclusions}

In order to create a robust manufacturing process for the fabrication of density engineered materials, the density gradient has to be measured, quantified and represented. These data will be feedback into the process to optimize the features of the tailor-made cellular structures. At present there is not a standard methodology for the characterization of density graded polymeric foams with open or closed cells. This is hindering a broader exploitation of functionally engineered materials in high technology applications such as aerospace or automotive, in optoelectronics, or in tissue engineering. This paper presents a novel characterization methodology for density engineered foams that were manufactured using the sonication process. This was achieved by the aggregation of a highly reliable data acquisition technique $(\mu-\mathrm{CT})$ and a robust image analysis software program (ImageJ).

The results derived from the cubic fitting coefficients, coefficients of skewness and global gradients 9 regions of interest (ROIs) were consistent with the visual inspection done on the foam samples.

The conclusions drawn from this study will inform the design and manufacturing process of density engineered materials used in other fields such as medicine (for the study of osteoporosis and the fabrication of implants), functionally tailored goods in the food \& drink industry, and in structural materials (e.g. packaging solutions and construction materials).

\section{Acknowledgements}


The authors would like to thank Dr Paul Tatum, at AWE plc, for allowing the acquisition of the

$\mu \mathrm{CT}$ images for the samples in this study; Dr Ruggero Gabbrielli for the photograph in Figure 1a and Dr Krassimir Dotchev for assistance in the fabrication of the sample at the Manufacturing Engineering Centre in Cardiff University; Dr Donald Fawcett, from Visuals Unlimited Inc. for Figure 1b. Dr Sarah Cartmell and Dr Lilia Araida Hidalgo-Bastida from Keele University kindly provided the images from the viability study in Figure 10.

\section{References}

[1] Schoonman A, Mayor G, Dillmann M-L, Bisperink C and Ubbink J 2001 The microstructure of foamed maltodextrin/sodium caseinate powders: a comparative study by microscopy and physical techniques Food Research International 34 913-29

[2] Willoughby A F W 2006 Design and processing of porous materials for electronic applications Philosophical Transactions of the Royal Society A: Mathematical, Physical and Engineering Sciences 364 175-87

[3] Kelly A 2006 Why engineer porous materials? Philosophical Transactions of the Royal Society A: Mathematical, Physical and Engineering Sciences 364 5-14

[4] Youn J R and Park H 1999 Bubble growth in reaction injection molded parts foamed by ultrasonic excitation Polymer Engineering and Science 39 457-68

[5] Yan X and Gu P 1996 A review of rapid prototyping technologies and systems Computer-Aided Design 28 307-18

[6] Dutta D, Prinz F B, Rosen D and Weiss L E 2001 Layered Manufacturing: Current status and future trends Journal of Computing and Information Science in Engineering 1 60-71

[7] Rajagopalan S and Robb R A 2006 Schwarz meets Schwann: Design and fabrication of biomorphic and durataxic tissue engineering scaffolds Medical Image Analysis 10 693-712

[8] Leong K F, Chua C K, Sudarmadji N and Yeong W Y 2008 Engineering functionally graded tissue engineering scaffolds Journal of the Mechanical Behavior of Biomedical Materials 1 140-52

[9] Woesz A, Rumpler M, Stampfl J, Varga F, Fratzl-Zelman N, Roschger P, Klaushofer K and Fratzl P 2005 Towards bone replacement materials from calcium phosphates via rapid prototyping and ceramic gelcasting Materials Science and Engineering: C 25 181-6

[10] Fukasawa T, Deng Z Y, Ando M, Ohji T and Goto Y 2001 Pore structure of porous ceramics synthesized from water-based slurry by freeze-dry process Journal of Materials Science 36 2523-7

[11] Deville S, Saiz E and Tomsia A P 2006 Freeze casting of hydroxyapatite scaffolds for bone tissue engineering Biomaterials 27 5480-9

[12] Sepulveda P and Binner J G P 1999 Processing of cellular ceramics by foaming and in situ polymerisation of organic monomers Journal of the European Ceramic Society 19 2059-66

[13] Rojas A J, Marciano J H and Williams R J 1982 Rigid polyurethane foams: A model of the foaming process Polymer Engineering \& Science $22840-4$ 
[14] Spoerke E D, Murray N G, Li H, Brinson L C, Dunand D C and Stupp S I 2005 A bioactive titanium foam scaffold for bone repair Acta Biomaterialia 1 523-33

[15] Kathuria Y P 2003 A preliminary study on laser assisted aluminum foaming Journal of Materials Science 38 2875-81

[16] Schwarzwalder K and Somers A V 1963 Method of making a porous shape of sintered refractory ceramic articles. (United States Patent Office: US3090094)

[17] Brothers A H and Dunand D C 2006 Density-graded cellular aluminum Advanced Engineering Materials 8 805-9

[18] Muhamad Nor M A A, Hong L C, Arifin Ahmad Z and Md Akil H 2008 Preparation and characterization of ceramic foam produced via polymeric foam replication method Journal of Materials Processing Technology 207 235-9

[19] Banhart J 2001 Manufacture, characterisation and application of cellular metals and metal foams Progress in Materials Science 46 559-632

[20] Mohamad Yunos D, Bretcanu O and Boccaccini A 2008 Polymerbioceramic composites for tissue engineering scaffolds Journal of Materials Science 43 4433-42

[21] Colombo P and Hellmann J 2002 Ceramic foams from preceramic polymers Materials Research Innovations 6 260-72

[22] Bil M, Ryszkowska J and Kurzydłowski K 2009 Effect of polyurethane composition and the fabrication process on scaffold properties Journal of Materials Science 44 1469-76

[23] Torres-Sánchez C and Corney J 2008 Effects of ultrasound on polymeric foam porosity Ultrasonics Sonochemistry 15 408-15

[24] Leighton T G 1995 Bubble population phenomena in acoustic cavitation Ultrasonics Sonochemistry 2 S123-S36

[25] Torres-Sánchez C and Corney J 2009 Identification of formation stages in a polymeric foam customised by sonication via electrical resistivity measurements Journal of Polymer Research 16 461-70

[26] Torres-Sanchez C and Corney J R 2009 Toward Functionally Graded Cellular Microstructures ASME Journal of Mechanical Design 131 910117

[27] Torres-Sanchez C and Corney J R 2009 Porosity tailoring mechanisms in sonicated polymeric foams IOP Journal of Smart Materials and Structures 18 104001-14

[28] Mullens S, Luyten J and Zeschky J 2005 Cellular Ceramics: Structure, Manufacturing, Properties and Applications, ed M Scheffler and $\mathrm{P}$ Colombo (Weinheim: Wiley-VCH Verlag GmbH \& Co., KGaA) pp 22566

[29] Malcolm A A, Leong H Y, Spowage A C and Shacklock A P 2007 Image segmentation and analysis for porosity measurement Journal of Materials Processing Technology 192 391-6

[30] Dobrzanski L A, Musztyfaga M, Grande M A and Rosso M 2009 Computer aided determination of porosity in sintered steels Archives of Materials Science and Engineering 38 103-11

[31] Torres-Sanchez C and Corney J R 2010 A novel manufacturing strategy for bio-inspired cellular structures International Journal of Design Engineering - Special issue "Design in nature" submitted

[32] Collins T J 2007 ImageJ for microscopy BioTechniques 43 S25-S30 
[33] Awcock G J and Thomas R 1996 Applied image processing: McGrawHill, Inc.)

Table:

Table 1: Cubic fitting coefficients for ROIs

\begin{tabular}{lllll}
\multicolumn{1}{c}{ ROIs } & $\mathbf{p}_{\mathbf{1}}(\mathbf{e}-\mathbf{0 7})$ & $\mathbf{p}_{\mathbf{2}}(\mathbf{e - 0 4})$ & $\mathbf{p}_{\mathbf{3}}$ & $\mathbf{p}_{\mathbf{4}}$ \\
ROI 1 & -2.6 & +2.2 & -0.07 & +16.0 \\
ROI 2 & -2.6 & +2.4 & -0.09 & +18.5 \\
ROI 3 & -2.6 & +2.2 & -0.07 & +18.0 \\
ROI 4 & -2.9 & +2.5 & -0.08 & +17.4 \\
ROI 5 & -3.3 & +2.8 & -0.09 & +18.3 \\
ROI 6 & -3.3 & +2.8 & -0.09 & +18.4 \\
ROI 7 & -3.6 & +3.5 & -0.11 & +19.2 \\
ROI 8 & -3.4 & +3.2 & -0.11 & +19.0 \\
ROI 9 & -3.5 & +3.3 & -0.11 & +20.0
\end{tabular}

Figures:

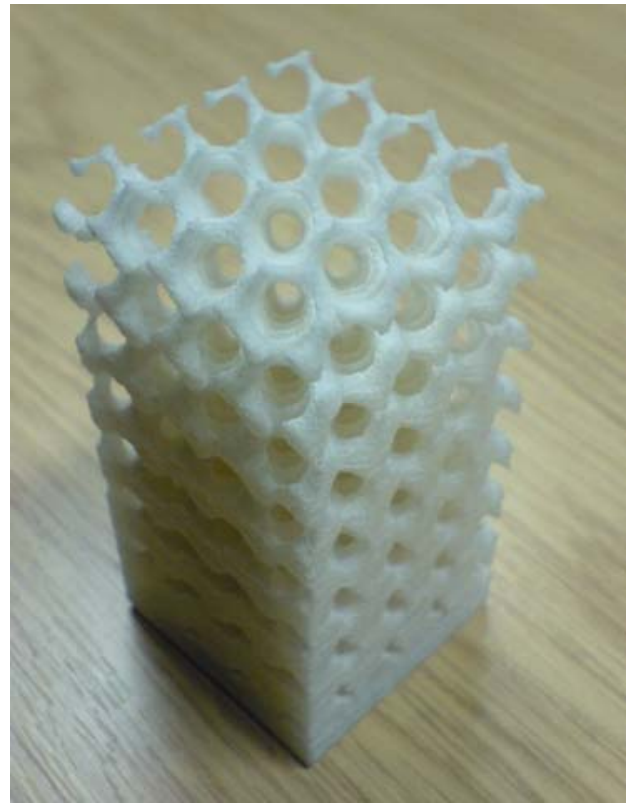

(a)

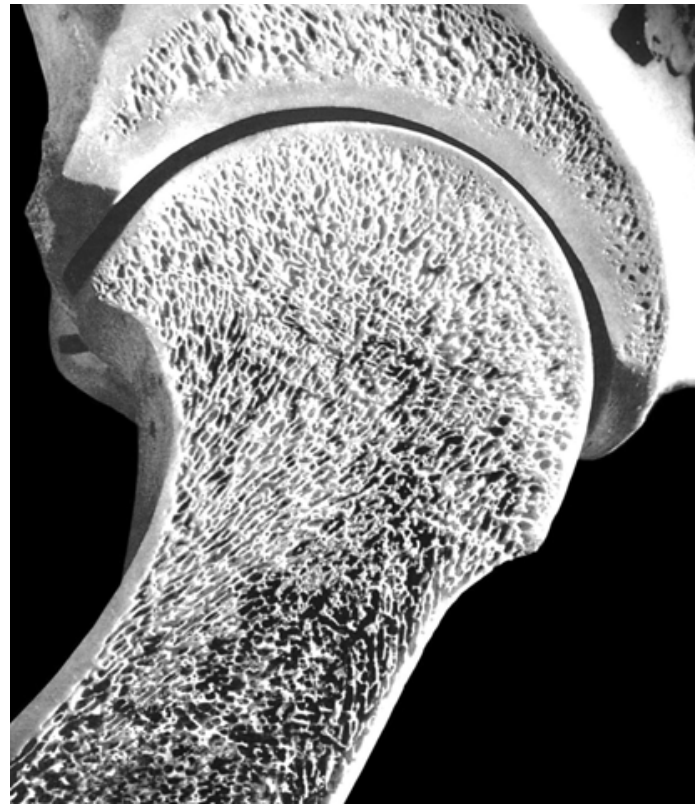

(b)

Figure 1: (a) 3D model fabricated by selective laser sintering in polyamide P12 showing the periodic cellular structure; (b) Longitudinal cross section of the human humerus bone showing the foam-like structure 


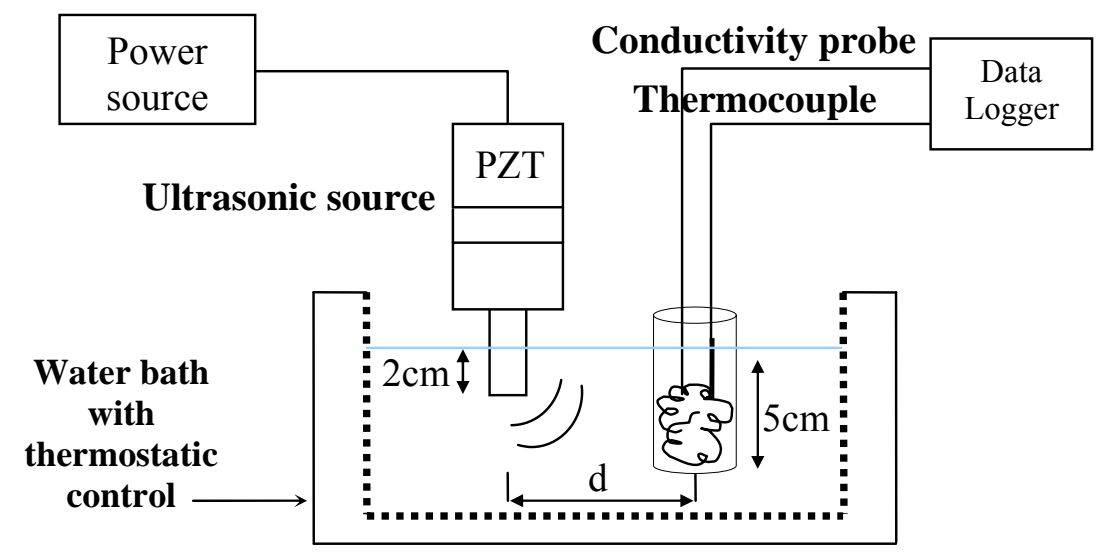

Figure 2: Schematic of the experimental rig

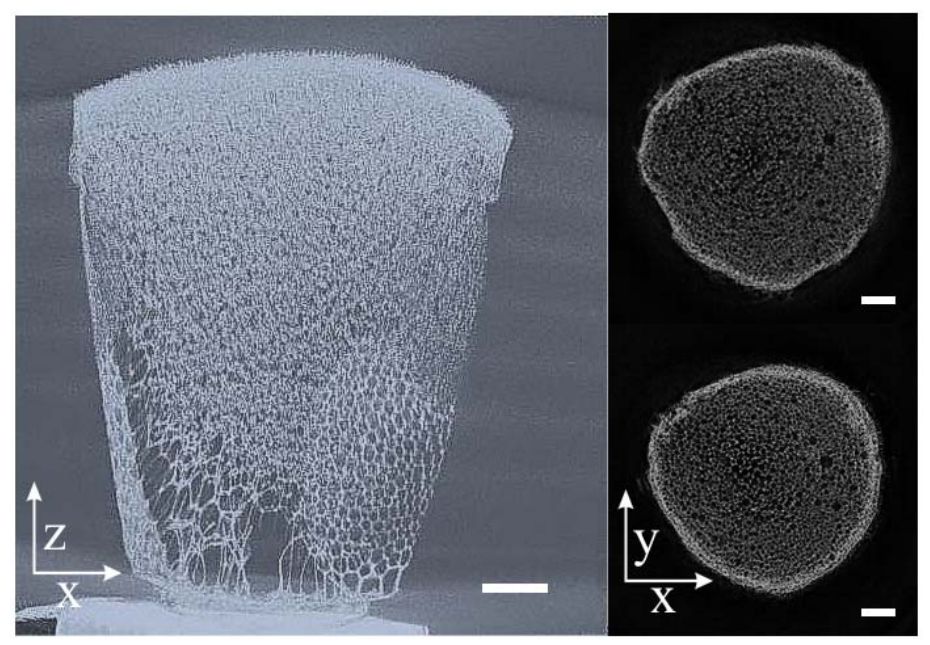

Figure 3: $\mu$-CT images of the foam sample. Diagonal cross-section showing the different pore sizes (left) and horizontal cross-sections at two different heights (right). Bars show $1 \mathrm{~cm}$ 


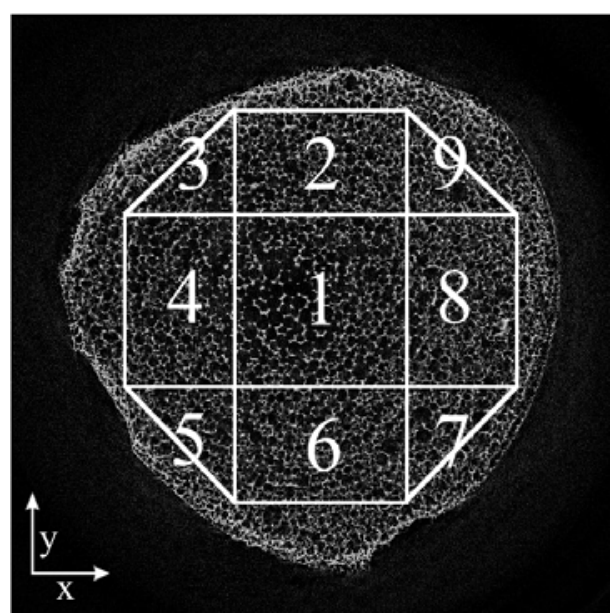

Figure 4: Regions of Interest (ROIs) on the foam cross-sections. These were kept fixed throughout the stack of images
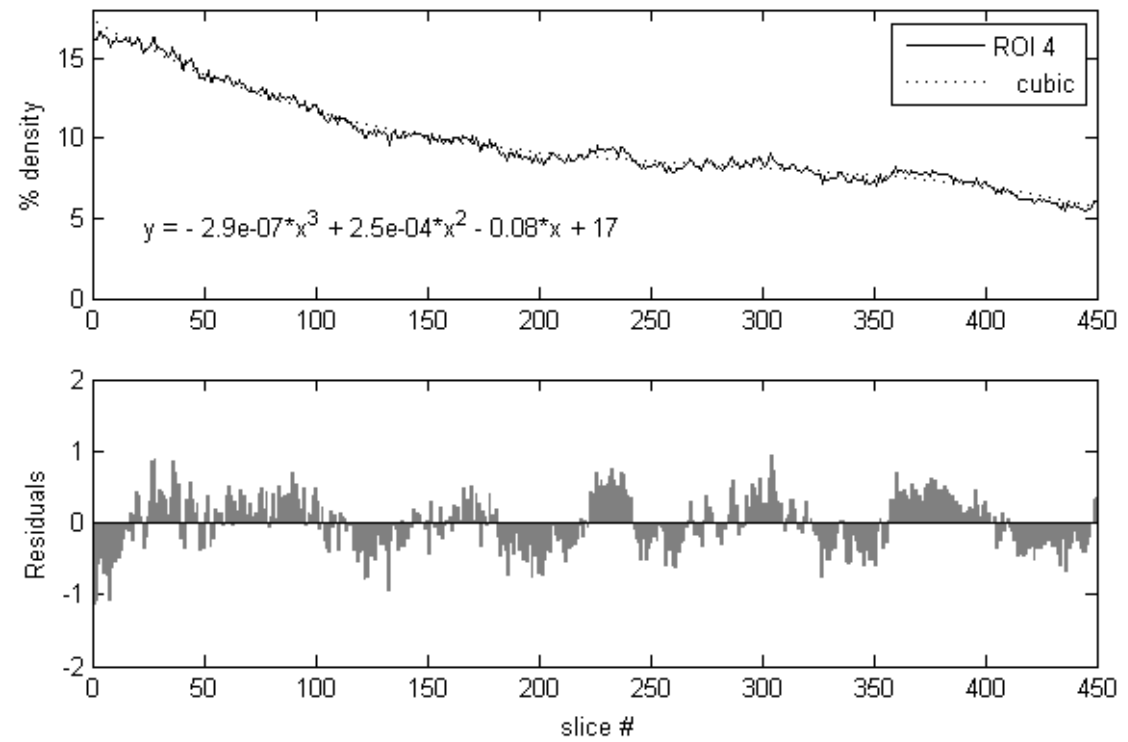

Figure 5: Density variation through the sample (vertically, from top to bottom) and residuals of the best fitting 


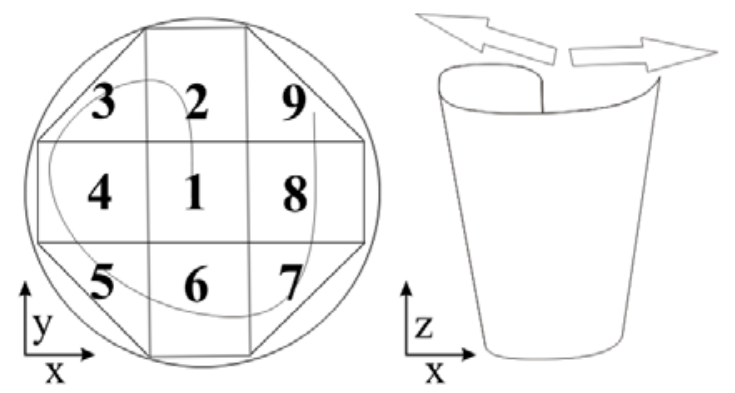

Figure 6: 'Unrolling' methodology for characterization of 3D density distribution

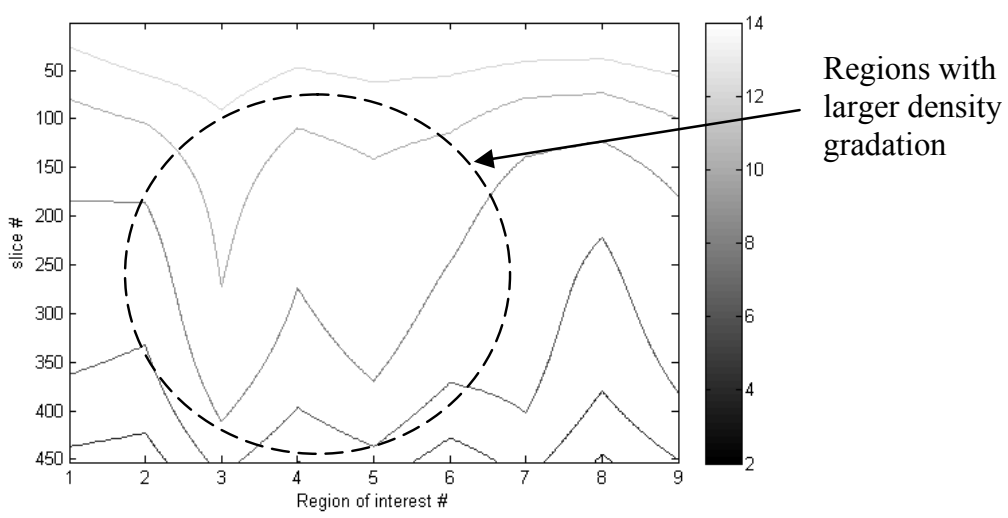

Figure 7: Global density distribution, plotted to show gradients. Density $2-14 \%$
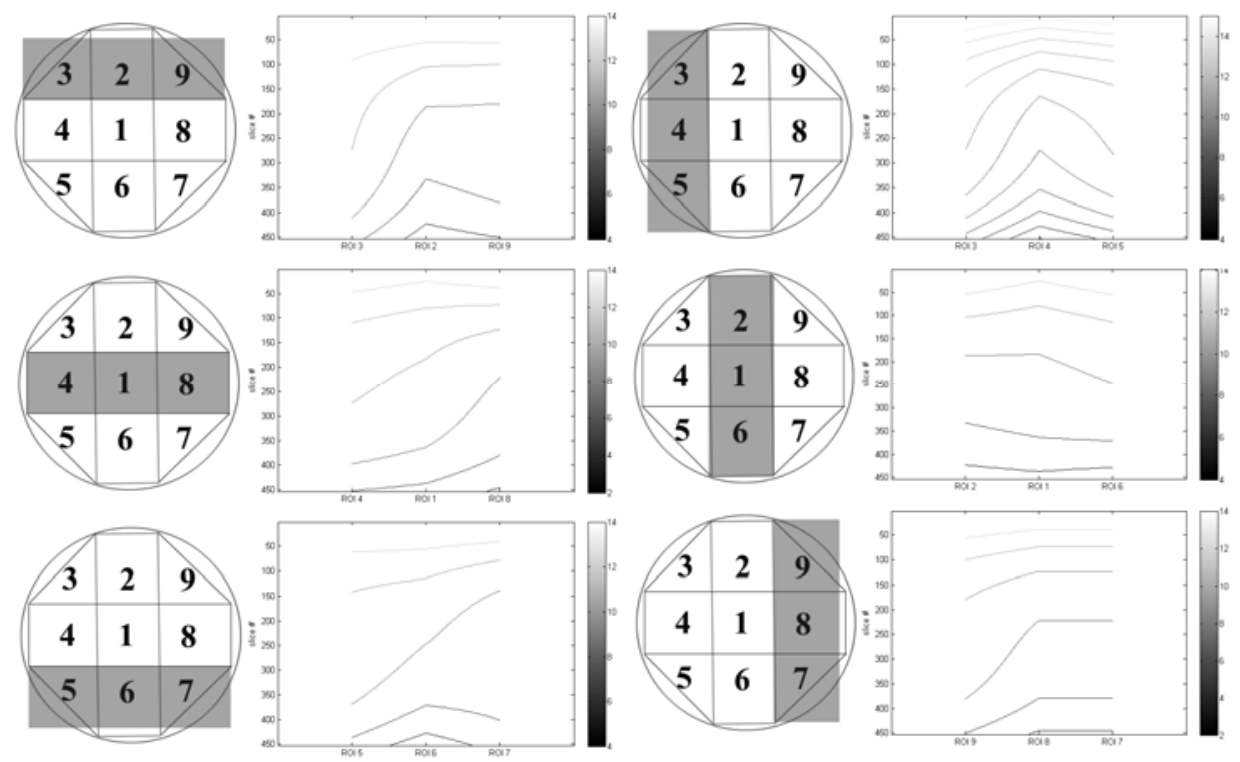

Figure 8: Density gradients for each of the ROIs. Shaded ROIs and correspondent density distribution values for each ROI 


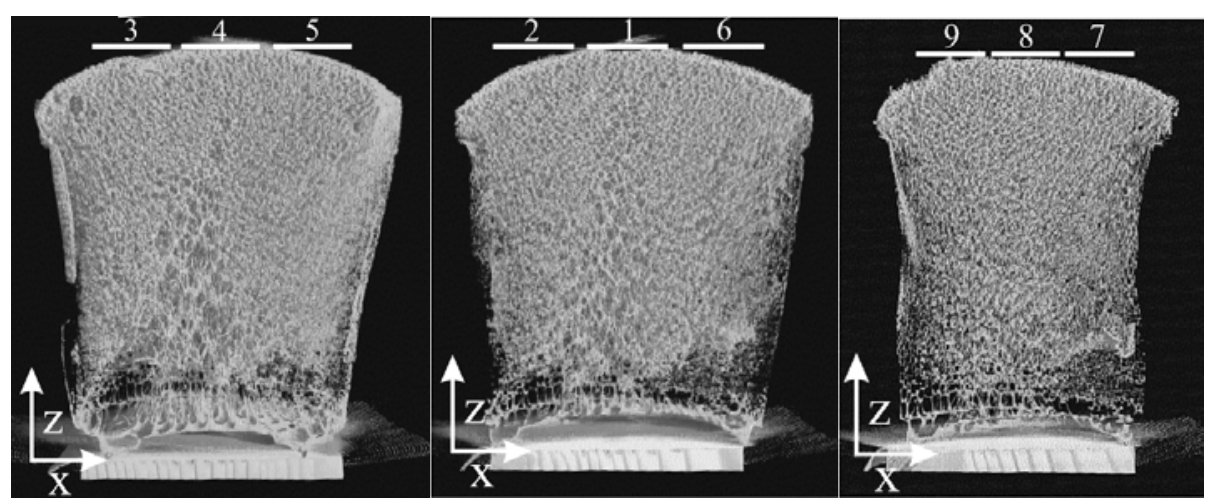

Figure 9: Visual inspection of the sample in three planes, show the different ROIs and the density gradients

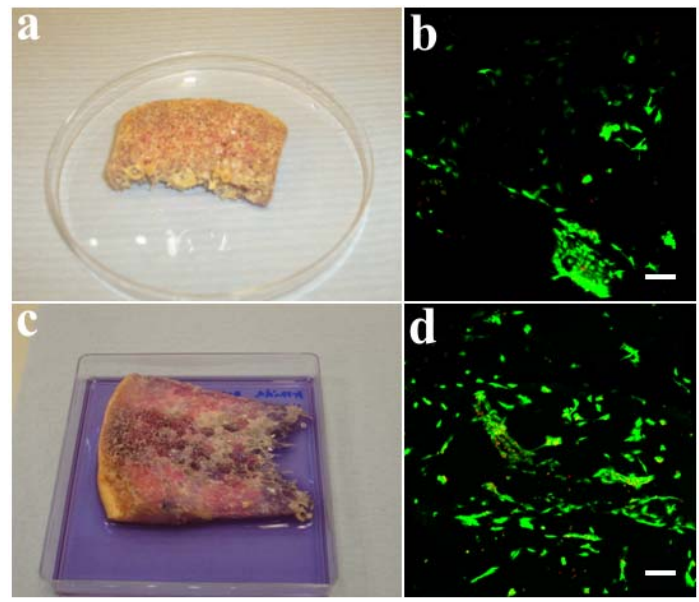

Figure 10: Cell viability study on PU porosity graded foams manufactured by traditional methods (top) and by sonication (bottom). The biocompatibility results show a higher viability for graded structures vs homogeneous textures. Bar shows $100 \mu \mathrm{m}$ 